TRANSACTIONS OF THE

AMERICAN MATHEMATICAL SOCIETY

Volume 190,1974

\title{
CLASS NUMBERS OF REAL QUADRATIC NUMBER FIELDS
}

\author{
BY
}

\section{EZRA BROWN}

ABSTRACT. This article is a study of congruence conditions, modulo powers of two, on class number of real quadratic number fields $Q(\sqrt{d})$, for which $d$ has at most three distinct prime divisors. Techniques used are those associated with Gaussian composition of binary quadratic forms.

1. Let $h(d)$ denote the class number of the quadratic field $Q(\sqrt{d})$ and let $b^{\prime \prime}(d)$ denote the number of classes of primitive binary quadratic forms of discriminant $d$ [if $d<0$ we count only positive forms]. It is well known [4] that $b(d)=$ $b^{\prime}(d)$, unless $d>0$ and the fundamental unit $\epsilon$ of $Q(\sqrt{d})$ has norm 1 , in which case $b(d)=1 / 2 b^{\prime}(d)$. Recently many authors have studied conditions on $d$ under which a given power of two divides $b(d)$ (see [3, References]). Most of these articles deal with imaginary fields; in this article, we shall treat real fields for which $d$ has at most three distinct prime divisors. Our method used to study this problem is the method of composition of forms, used in [1], [3]; we have included several known cases for the sake of completeness.

2. Preliminaries. A binary quadratic form is called ambiguous if its square, under Gaussian composition, is in the principal class, i.e. the class representing 1 (see [1] for explanations of any unfamiliar terminology). A class of forms is called ambiguous if it contains an ambiguous form. A form $[a, b, c]=a x^{2}+b x y+$ $c y^{2}$ is called ancipital if $b=0$ or $b=a$. It was known to Gauss that the number of ambiguous classes of discriminant $d$ equals the number of genera of discriminant $d$ (see [7]), and that each ambiguous class of positive nonsquare discriminant contains exactly two ancipital forms with positive first coefficient (see [7]).

The primitive forms of discriminant $d$ form an abelian group $G$ of order $b^{\prime}(d)$, the operation being composition and the identity being the principal class I. The principal genus $G^{+}$is a subgroup of $G$ consisting of all the classes which are squares under composition; the index of $G^{+}$in $G$ equals the number of genera. If $d$ is the discriminant of a quadratic field, then $d$ is fundamental, i.e. no square $s^{2}>1$ exists for which $d / s^{2} \equiv 0$ or $1(\bmod 4)$. Hence the number of genera

Received by the editors October 3, 1972.

AMS (MOS) subject classifications (1970). Primary 10A15, 10C05, 12A25, $12 \AA 50$.

Key words and phrases. Class number, quadratic residues, quadratic fields, algebraic number fields, Gaussian composition, binary quadratic forms. 
of a fundamental discriminant $d$ is equal to $2^{\gamma+\theta-1}$, where $\gamma$ is the number of distinct odd primes dividing $d$ and $\theta=0$ or 1 according as $d \equiv 1(\bmod 4)$ or $d \equiv 8,12(\bmod 16)$. (For proofs of any of the above statements, see [5].) Throughout this paper $p, q$ and $p_{i}$ denote odd primes and $d$ is a fundamental discriminant; $(p \mid q)$ is the Legendre symbol, and we write $(p \mid q)_{4}=1$ or -1 according as $p$ is or is not a biquadratic residue of $q$. If $f$ and $g$ are forms we write $f \sim g$ or $f \chi_{g}$ according as $f$ is or is not equivalent to $g$.

3. Discriminants with one prime divisor. If $d$ is a positive discriminant with exactly one prime divisor, then there is only one genus; this genus contains the one ambiguous class, namely $I$, and several pairs of improperly equivalent nonambiguous classes. Thus, $b^{\prime}(d)$ is odd; it is impossible that $b(d)=1 / 2 b^{\prime}(d)$, so that $b(d)=b^{\prime}(d)$, the norm $N \epsilon$ of the fundamental unit is -1 , and $b(d)$ is odd.

4. The case $d=4 p$ or $d=8 p$. If $d=4 p$ is fundamental, then $p \equiv 3(\bmod 4)$; there are two genera, hence two ambiguous classes. Since $N \epsilon=+1$, the ambiguous classes are $I$ and $-I$, and $-I$ contains the form $f_{-1}=[p, 0,-1]$; since $\left(f_{-1} \mid p\right)=$ $-1,-I \notin G^{+}$. Hence each genus contains an ambiguous class and several pairs of improperly equivalent nonambiguous classes. Since each genus always contains the same number of classes, we deduce that $b^{\prime}(4 p) \equiv 2(\bmod 4)$ and hence $b(4 p)$ is odd. Similarly, if $d=8 p$ and $p \equiv 3(\bmod 4)$, there are two genera; the ambiguous classes are $I$ and $-I$, distributed one to each genus. Hence $b^{\prime}(8 p) \equiv 2(\bmod 4)$ and $b(8 p)$ is odd.

Let $d=8 p$ with $p \equiv 1(\bmod 4)$. The four ancipital forms with positive first coefficient are $f_{1}=[1,0,-2 p], f_{-1}=[2 p, 0,-1], f_{2}=[2,0,-p]$ and $f_{-2}=$ $[p, 0,-2]$, and the generic invariants are $(m \mid p)$ and $(2 \mid m)$. If $p \equiv 5(\bmod 8)$, then $\left(f_{2} \mid p\right)=\left(f_{-2} \mid p\right)=-1$, so that $f_{2} \notin G^{+}$and the ambiguous classes are distributed one to each genus. As above, we deduce that $b^{\prime}(8 p)=2(\bmod 4) ;$ however, $f_{2} \notin G^{+}$ imply that $f_{1} \sim f_{-1}$, so that $N \epsilon=-1$. Hence $b(8 p)=b^{\prime}(8 p) \equiv 2(\bmod 4)$.

Let $d=8 p, p \equiv 1(\bmod 8)$. Then both ambiguous classes lie in the principal genus. Denote by $N$ the nonprincipal ambiguous class: since $N \in G^{+}, N=D^{2}$ for some class $D$, and thus $D$ has order 4 in $G$. If we let $H=\left\{D, N=D^{2}, D^{3}, l=D^{4}\right\}$, then $b^{\prime}(8 p)=$ ord $G=\operatorname{ord}(G / H) \cdot$ ord $H \equiv 0(\bmod 4)$. Now $p \equiv 1(\bmod 8)$ so there exist positive integers $a, b, e$ and $f$ such that $p=a^{2}+2 b^{2}=2 f^{2}-e^{2}$. Let $g_{-2}=$ $[a, 4 b,-2 a]$ and let $g_{2}=[e, 4 f, 2 e]$; then $g_{-2}^{2} \sim f_{-2}$ and $g_{2}^{2} \sim f_{2}$. It is clear that $g_{-2}$ has order 4 whenever $f_{-2} \in N$ and $g_{2}$ has order 4 whenever $f_{2} \in N$. Hence $g_{-2} \in D$ or $D^{3}$ if $f_{-2} \in N$, and $g_{2} \in D$ or $D^{3}$ if $f_{2} \in N$. If $D \in G^{+}$, then $b^{\prime}(8 p) \equiv 0(\bmod 8)$, since $b^{-}(8 p)=\operatorname{ord}\left(G / G^{+}\right) \cdot \operatorname{ord}\left(G^{+} / H\right) \cdot \operatorname{ord} H=2 \cdot \operatorname{ord}\left(G^{+} H\right)$. $4 \equiv 0(\bmod 8)$. If $D \notin G^{+}$, let $B$ be a class for which $B^{2} \in H$. Now $B^{2} \neq D$ or $D^{3}$, since $D$ and $D^{3}$ are not squares. If $B^{2}=N$, then $B^{2} D^{2}=N^{2}=I$, so that $B D=I$ or $N$, i.e., $B=D^{3}$ or $D$. If $B^{2}=I$, then $B=N$ or $I$. Hence the index of $H$ in $G$ is odd i.e. $b^{\prime}(8 p)=\operatorname{ord}(G / H) \cdot \operatorname{ord} H \equiv 4(\bmod 8)$. 
Now $g_{-2} \in G^{+}$if and only if $a \equiv \pm 1(\bmod 8)$ and $g_{2} \in G^{+}$if and only if $e \equiv \pm 1(\bmod 8)$. In addition, $\pm e \equiv 1$ or $3(\bmod 8)$ according as $p \equiv 1$ or $9(\bmod 16)$.

Case (a): $p \equiv 9(\bmod 16)$. By Pall [7], $f_{1} \sim f_{-1}$ if and only if $b \equiv 0(\bmod 4)$ and $f_{1} \sim f_{-2}$ if and only if $b \equiv 2(\bmod 4)$. Hence, $f_{2} \in N$ and $g_{2} \in D$; but $\pm e \equiv 3(\bmod 8)$, so that $D \notin G^{+}$. Thus, if $b \equiv 0(\bmod 4)$, then $N \epsilon=-1$ and $b(8 p)=$ $b^{\prime}(8 p) \equiv 4(\bmod 8) ;$ if $b \equiv 2(\bmod 4)$, then $N \epsilon=+1$ and $b(8 p)=1 / 2 b^{\prime}(8 p) \equiv 2(\bmod 4)$.

Case (b): $p \equiv 1(\bmod 16)$. Now $\pm a \equiv 1$ or $3(\bmod 8)$ according as $b \equiv 0$ or $2(\bmod 4)$. By Pall [7], if $b \equiv 2(\bmod 4)$, then $f_{1} \sim f_{2} ;$ in that case, $f_{-2} \in N$, $g_{-2} \in D$ and $\pm a \equiv 3(\bmod 8)$, so that $D \notin G^{+}$and $b^{\prime}(8 p) \equiv 4(\bmod 8)$. Since $f_{1} x f_{-1}, b(8 p) \equiv 2(\bmod 4)$. If $b \equiv 0(\bmod 4)$, any of $f_{-1}, f_{2}$ and $f_{-2}$ may be in $I$ : since $\pm a \equiv 1$ and $\pm e \equiv 1(\bmod 8), D \in G^{+}$and $b^{\prime}(8 p) \equiv 0(\bmod 8)$. Hence $b(8 p) \equiv$ $0(\bmod 4)$ unless $f_{1} \sim f_{-1}$, in which case $b(8 p) \equiv 0(\bmod 8)$.

Thus we have proved the following theorem.

Theorem 1. Let $p$ be an odd prime.

(a) If $p \equiv 3(\bmod 4)$, then $b^{\prime}(4 p) \equiv b^{\prime}(8 p) \equiv 2(\bmod 4)$ and $b(4 p) \equiv b(8 p) \equiv$ $1(\bmod 2)$.

(b) If $p \equiv 5(\bmod 8)$, then $b(8 p)=b^{\prime}(8 p) \equiv 2(\bmod 4)$.

(c) If $p \equiv 1(\bmod 8)$, write $p=a^{2}+2 b^{2}$ with $a$ and $b$ positive integers. If $p \equiv 1(\bmod 16)$ and $b \equiv 0(\bmod 4)$, then $b^{\prime}(8 p) \equiv 0(\bmod 8) ;$ otberwise, $b^{\prime}(8 p) \equiv$ $4(\bmod 8)$.

(d) If $p \equiv 9(\bmod 16)$, then $b(8 p) \equiv 4(\bmod 8)$ or $b(8 p) \equiv 2(\bmod 4)$ according as $b \equiv 0$ or $2(\bmod 4)$.

(e) If $p \equiv 1(\bmod 16)$, then $b(8 p) \equiv 2(\bmod 4)$ if $b \equiv 2(\bmod 4)$; bowever, if $b \equiv 0(\bmod 4)$, then $b(8 p) \equiv 0(\bmod 4$ or 8$)$ according as $x^{2}-2 p y^{2}=-1$ does not or does bave a solution in integers.

Remark. If $f_{-2}$ is a fourth power, then $g_{-2} \in G^{+}$, i.e. $(a \mid p)=(2 \mid a)=1$. Since $f_{-2} \sim g_{-2}^{2}$, we may write $a^{2}=p x^{2}-2 y^{2}$ where $x$ is odd, $y=2^{a} y^{\prime}, a>1$ and $y^{\prime}$ is odd. Hence we have that

$$
(2 \mid a)=(a \mid p)=(-1 \mid p)_{4}(2 \mid p)_{4}(2 \mid p)^{a}\left(y^{\prime} \mid p\right)=(2 \mid p)_{4},
$$

since $p \equiv 1(\bmod 8)$ and $\left(p \mid y^{\prime}\right)=1$. Thus $f_{-2}$ is a fourth power if and only if $(2 \mid p)_{4}(=(2 \mid a))=1$. It is then straightforward to verify that

$$
(2 \mid p)_{4}=(-1)^{(p-1) / 8+b / 2}
$$

which is one form of a result due to Dirichlet [7].

5. The case $d=p q, p \equiv q(\bmod 4)$. We shall prove the following:

Theorem 2. Let $p \equiv q(\bmod 4)$ be distinct odd primes. The congruence conditions on $b(p q)$ and $b^{\prime}(p q)$ modulo powers of 2 are given by the following table. 


$$
\begin{array}{ccc}
p \text { and } q & b^{\prime}(p q)(\bmod m) & b(p q)(\bmod m) \\
p \equiv q \equiv 3(\bmod 4) & 2(\bmod 4) & 1(\bmod 2) \\
p \equiv q \equiv 1(\bmod 4): & 2(\bmod 4) & 2(\bmod 4) \\
(p \mid q)=-1 & \\
(p \mid q)=1: & \\
(p \mid q)_{4}=(q \mid p)_{4}=-1 & 4(\bmod 8) & 4(\bmod 8) \\
(p \mid q)_{4}(q \mid p)_{4}=-1 & 4(\bmod 8) & 2(\bmod 4) \\
(p \mid q)_{4}=(q \mid p)_{4}=1 & 0(\bmod 8) & 0(\bmod 8) \text { if } N \epsilon=-1 ; \\
& & 0(\bmod 4) \text { if } N \epsilon=1 .
\end{array}
$$

Proof. It is known [6] that $b^{\prime}(p q)$ and $b^{\prime}(4 p q)$ are exactly divisible by the same power of 2 if $p \equiv q(\bmod 4)$; the forms of discriminant $4 p q$ are easier to treat, so we will use them to get results on discriminant $p q$. There are two genera, hence two ambiguous classes, and the four ancipital forms with positive first coefficients are $f_{1}=[1,0,-p q], f_{-1}=[p q, 0,-1], f_{p}=[p, 0,-q]$ and $f_{-p}=[q, 0,-p]$.

If $p \equiv q \equiv 3(\bmod 4)$, then $f_{-1} \notin G^{+}$, and if $p \equiv q \equiv 1(\bmod 4)$ and $(p \mid q)=-1$, then $f_{1} \sim f_{-1}$ and $f_{p} \notin G^{+}$. Hence the ambiguous classes are distributed one to a genus and we have $b^{\prime}(p q) \equiv 2(\bmod 4)$. If $p \equiv q \equiv 3(\bmod 4)$, then $f_{-1} \not f_{1}$, so that $b(p q)$ is odd; if $p \equiv q \equiv 1(\bmod 4)$ and $(p \mid q)=-1$, then $b(p q)=b^{\prime}(p q) \equiv 2(\bmod 4)$.

If $p \equiv q \equiv 1(\bmod 4)$ and $(p \mid q)=1$, then both ambiguous classes are in the principal genus, so that $b^{\prime}(p q) \equiv 0(\bmod 4)$. Now $f_{p} \in G^{+}$, so there is a form $g_{p}$ with leading coefficient $r$ prime to $2 p q$ such that $f_{p} \sim g_{p}^{2}$, whence $f_{p}$ represents $r^{2}$. Hence $f_{p}$ is a fourth power exactly when $(r \mid p)=(r \mid q)=1$. Writing $r^{2}=p x^{2}$ $-q y^{2}$, we see that $x$ is odd, $y=2^{\alpha} y^{\prime}, \alpha>0, y^{\prime}$ is odd and $\alpha=1$ if and only if $p \equiv 5(\bmod 8) ;$ furthermore, $\left(p \mid y^{\prime}\right)=1$ and $(-1 \mid p)_{4}=(2 \mid p)$ whenever $p \equiv 1(\bmod 4)$. Hence $(r \mid p)=(q \mid p)_{4}(2 \mid p)^{a+1}\left(y^{\prime} \mid p\right)=(q \mid p)_{4}$, and hence $f_{p}$ is a fourth power if and only if $(q \mid p)_{4}=1$. Similarly, $f_{-p}$ is a fourth power if and only if $(p \mid q)_{4}=1$. Since $b^{\prime}(p q) \equiv 0(\bmod 4), f_{1}$ is a fourth power, which implies that $N \epsilon=-1$ if $(p \mid q)_{4}=(q \mid p)_{4}=-1$.

If $(p \mid q)_{4}(q \mid p)_{4}=-1$, then exactly one of $f_{p}$ and $f_{-p}$ is a fourth power, that one is in $l$, and hence $f_{-1} \notin l$. Thus, $f_{1} \sim f_{p}$ if $(q \mid p)_{4}=1=-(p \mid q)_{4}$, and $f_{1} \sim f_{-p}$ if $(p \mid q)_{4}=1=-(q \mid p)_{4}$ (for an alternate proof of this, see [2]).

Furthermore, if $(p \mid q)_{4}=-1$ or $(q \mid p)_{4}=-1$, then $b^{\prime}(p q) \equiv 4(\bmod 8)$. To see this, let $D$ be a class such that $D^{2}=N$, the nonprincipal ambiguous class. If $(p \mid q)_{4}=-1$ or $(q \mid p)_{4}=-1$, then $D \notin G^{+}$by the above, so that neither $D$ nor $D^{3}$ 
is a square. As in the proof of Theorem 1, it is straightforward to verify that $H=\left\{I, D, D^{2}, D^{3}\right\}$ has odd index in $G$, i.e. $b^{\prime}(p q) \equiv 4(\bmod 8)$.

Finally, if $(p \mid q)_{4}=(q \mid p)_{4}=1$, then $H \subseteq G^{+}$, so that $b^{\prime}(p q) \equiv$ ord $G \equiv$ $\operatorname{ord}\left(G / G^{+}\right) \cdot \operatorname{ord}\left(G^{+} / H\right) \cdot \operatorname{ord} H \equiv 2 \cdot \operatorname{ord}\left(G^{+} / H\right) \cdot 4 \equiv 0(\bmod 8)$. Hence $b(p q) \equiv$ $0(\bmod 8)$ if $N \epsilon=-1$ and $b(p q) \equiv 0(\bmod 4)$ if $N \epsilon=1$. We are done.

Remark. There still remains open the difficult case $(p \mid q)_{4}=(q \mid p)_{4}=1$. One possible approach is the following: since $b^{\prime}(p q) \equiv 0(\bmod 8), D \in G^{+}$, so that $D$ represents an odd square $r^{2}$ prime to $p q$, where $r$ is represented by $B$ and $B^{2}=D$. Then it is easily shown that $b^{\prime}(p q) \equiv 0(\bmod 16)$ if and only if $B \in G^{+}$, i.e. $(r \mid p)=$ $(r \mid q)=1$. Now we have $r^{2}=k x^{2}+2 m x y+n y^{2}$, where $[k, 2 m, n] \in D$; perhaps examining this equation modulo $p$ would yield results. A similar remark applies to the case $p \equiv 1(\bmod 16)$ and $b \equiv 0(\bmod 4)$ for discriminants $d=8 p$.

6. The case $d=4 p q, p \equiv q(\bmod 4)$. Let $p \equiv 1 \equiv-q(\bmod 4)$. There are four ambiguous classes: Since $d \equiv 12(\bmod 16)$, the generic characters are $(m \mid p)$, $(m \mid q)$ and $(-1 \mid m)$. Since $f_{-1}=[p \dot{q}, 0,-1]$ satisfies $\left(-1 \mid f_{1}\right)=-1, f_{-1} \notin G^{+}$, so that $N \epsilon=+1$ and $b(4 p q)=1 / 2 b^{\prime}(4 p q)$. The other ancipital forms are $f_{1}, f_{p}$ and $f_{-p}$ of $\$ 5$, and $f_{2}=[2,2,1 / 2(1-p q)], f_{-2}=[1 / 2(p q-1),-2,-2], f_{2 p}=$ $[2 p, 2 p, 1 / 2(p-q)]$ and $f_{2 q}=[2 q, 2 q, 1 / 2(q-p)]$. We construct a table of generic characters (from the Gauss product relation, we know that $(-1 \mid m)=(m \mid p)(m \mid q)$ ):

$\begin{array}{lcc} & (m \mid p) & (m \mid q) \\ f_{1} & 1 & 1 \\ f_{-1} & 1 & -1 \\ f_{p} & (p \mid q) & (p \mid q) \\ f_{-p} & (p \mid q) & -(p \mid q) \\ f_{2} & (2 \mid p) & (2 \mid q) \\ f_{-2} & (2 \mid p) & -(2 \mid q) \\ f_{2 p} & (2 \mid p)(p \mid q) & (2 \mid q)(p \mid q) \\ f_{2 q} & (2 \mid p)(p \mid q) & -(2 \mid q)(p \mid q)\end{array}$

From this table, it is evident that if $(p \mid q)=1$, then $f_{1}, f_{-1}, f_{p}$ and $f_{-p}$ are all in different genera; in addition, if $p \equiv 5(\bmod 8)$, then $f_{1}, f_{-1}, f_{2}$ and $f_{-2}$ are all in different genera. In those cases, each of the four genera contains an odd number of classes: hence $b^{\prime}(4 p q)=4(\bmod 8)$ and $b(4 p q)=2(\bmod 4)$.

Let $p \equiv 1(\bmod 8)$ and $(p \mid q)=1$. Then $f_{1}$ and $f_{p} \in G^{+} ;$in addition, $f_{-2}$ and $f_{2 q} \in G^{+}$if $q \equiv 3(\bmod 8)$, and $f_{2}$ and $f_{2 p} \in G^{+}$if $q \equiv 7(\bmod 8)$. Thus $G^{+-2}$ has even order and hence $b^{\prime}(4 p q)=\operatorname{ord} G^{+} \cdot \operatorname{ord}\left(G / G^{+}\right) \equiv 0(\bmod 8)$. If $f_{p}$ is to be a 
fourth power, then there exists a form $g_{p}$ such that $f_{p} \sim g_{p}^{2}$ and $g_{p} \in G^{+}$: this can happen if and only if $(r \mid p)=(r \mid q)=(-1 \mid r)=1$, where $r$ is primitively represented by $g_{p}$. If $r$ is such a number, we may write $r^{2}=p x^{2}-q y^{2}$, where $y=2^{a} y^{\prime}$ and $y^{\prime}$ is odd: then $(r \mid p)=(-1 \mid p)_{4}(q \mid p)_{4}(2 \mid p)^{a}\left(y^{\prime} \mid p\right)=(q \mid p)_{4}$. [Note: We cannot do this $\bmod q$ since $(m \mid q)_{4}=(m \mid q)$; also, the condition $(-1 \mid r)=1$ is of no use.] Hence, if $f_{p}$ is a fourth power, then $(q \mid p)_{4}=1$; thus, if $p \equiv 1(\bmod 8),(p \mid q)=1$ and $(q \mid p)_{4}=-1$, then $f_{1} \chi f_{p}$, since $f_{1}$ is always a fourth power; by the grouptheoretic arguments previously used, we deduce that $b^{\prime}(4 p q) \equiv 8(\bmod 16)$.

Let $q \equiv 3(\bmod 8)$ and write $p q=f^{2}+2 e^{2}$. The form $g_{-2}=[e, 2 f,-2 e]$ satisfies $g_{-2}^{2} \sim f_{-2}$. Hence $f_{-2}$ is a fourth power if and only if $(e \mid p)=(-1 \mid e)=1$ (note that $(p \mid q)=(e \mid p)(-1 \mid e))$. Since $[-e, 2 f,-2(-e)]^{2} \sim f_{-2}$, we may assume that $e \equiv 1(\bmod 4)$. Thus, $f_{-2}$ is a fourth power if and only if $p q=f^{2}+2 e^{2}$, where $e \equiv 1(\bmod 4)$ and $(e \mid p)=1$. Similarly, if $q \equiv 7(\bmod 8)$, it can be shown that $f_{2}$ is a fourth power if and only if $p q=f^{2}-2 e^{2}$ where $e \equiv 1(\bmod 4)$ and $(e \mid p)=1$. Thus, by the group-theoretic arguments previously used, we deduce that if $q \equiv$ $3(7)(\bmod 8)$ and $f_{1} \chi f_{-2}\left(f_{2}\right)$, then $b^{\prime}(4 p q) \equiv 0$ or $8(\bmod 16)$ according as $p q=$ $f^{2}+2 e^{2}\left(p q=f^{2}-2 e^{2}\right)$ with $(e \mid p) \equiv 1 \equiv e(\bmod 4)$ or not. Finally, we observe that $f_{1} \sim f_{p}$ or $f_{1} \sim f_{ \pm 2}$, but not both. We thus have the following theorem.

Theorem 3. (a) Let $p \equiv 1 \equiv-q(\bmod 4)$ be primes; then $b(4 p q)=1 / 2 b^{\prime}(4 p q)$. If $p \equiv 5(\bmod 8)$ or if $(p \mid q)=-1$, then $b^{\prime}(4 p q) \equiv 4(\bmod 8)$; otherwise $b^{\prime}(4 p q) \equiv$ $0(\bmod 8)$.

(b) Let $p \equiv 1, q \equiv 3(7)(\bmod 8)$ and $(p \mid q)=1$; write $p q=f^{2}+2 e^{2}\left(f^{2}-2 e^{2}\right)$ with $e \equiv 1(\bmod 4)$. If $(e \mid p)=-1$ or if $(q \mid p)_{4}=-1$, then $b^{\prime}(4 p q) \equiv 8(\bmod 16)$. If $x^{2}-p q y^{2}=-2(+2)$ bas no solutions and $(e \mid p)=1$, then $b^{\prime}(4 p q) \equiv 0(\bmod 16)$.

7. The case $d=8 p q$. There are four genera and the eight ancipital forms with positive first coefficients are as follows:

$$
\begin{array}{ll}
f_{1}=[1,0,-2 p q], & f_{-1}=[2 p q, 0,-1], \\
f_{2}=[2,0,-p q], & f_{-2}=[p q, 0,-2], \\
f_{p}=[p, 0,-2 q], & f_{-p}=[2 q, 0,-p], \\
f_{q}=[q, 0,-2 p], & f_{-q}=[2 p, 0,-q] .
\end{array}
$$

If we construct a table of generic characters, we then deduce the following information: 


\begin{tabular}{lcll}
$p(\bmod 8)$ & $q(\bmod 8)$ & \multicolumn{2}{c}{ Ancipital forms $f_{i}$ in $G^{+}$} \\
& & $(p \mid q)=-1$ & $(p \mid q)=1$ \\
1 & 1 & $f_{ \pm 1}, f_{ \pm 2}$ & All \\
1 & 5 & $f_{ \pm 1}$ & $f_{ \pm 1}, f_{ \pm p}$ \\
5 & 5 & $f_{ \pm 1}$ & $f_{ \pm 1}$ \\
3 & 3 & $f_{1}, f_{-2}$ & $f_{1}, f_{-2}$ \\
3 & 7 & $f_{1}, f_{q}$ & $f_{1}, f_{-q}$ \\
7 & 7 & $f_{1}, f_{2}, f_{-p}, f_{q} f_{1}, f_{2}, f_{-p}, f_{-q}$ \\
1 & 3 & $f_{1}, f_{-2}$ & $f_{1}, f_{-2}, f_{p}, f_{q}$ \\
1 & 7 & $f_{1}, f_{2}$ & $f_{1}, f_{2}, f_{p}, f_{-q}$ \\
5 & 3 & $f_{1}, f_{-p}$ & $f_{1}, f_{q}$ \\
5 & 7 & $f_{1}, f_{-p}$ & $f_{1}, f_{-q}$
\end{tabular}

From this we observe that $G^{+}$contains all four ambiguous classes if $(p \mid q)=1 \equiv$ $p \equiv q(\bmod 8) ;$ two ambiguous classes if (a) $p \equiv q \equiv 7(\bmod 8),($ b) $p \equiv q \equiv 1(\bmod 8)$ and $(p \mid q)=-1$, or $(c) p \equiv 1 \not \equiv q(\bmod 8)$ and $(p \mid q)=1$; one ambiguous class in all other cases. By using the same techniques as we used in the previous sections we may prove the following lemma (the painful but elementary details are omitted).

Lemma. (1) Let $p \equiv 1(\bmod 8)$ and suppose $(p \mid q)=1$. If $f_{p}$ is a fourth power, then $(2 \mid p)_{4}=(q \mid p)_{4}$.

(2) Let $p \equiv 1(\bmod 8), q \equiv 1(\bmod 4)$ and $(p \mid q)=1$. If $f_{-p}$ is a fourth power, then $(p \mid q)_{4}=1$ or -1 according as $p \equiv 1$ or $9(\bmod 16)$.

(3) Let $p \equiv 1$ and $q \equiv 1$ or $3(\bmod 8)$; write $p q=e^{2}+2 f^{2}$ with $e \equiv 1(\bmod 4)$. Then $f_{-2}$ is a fourtb power if and only if $(e \mid p)=(e \mid q)=1$.

(4) Let $p \equiv 1 \equiv \pm q(\bmod 8)$; write $p q=2 f^{2}-e^{2}$ with $e \equiv 1(\bmod 4)$. Then $f_{2}$ is a fourth power if and only if $(e \mid p)=(e \mid q)=1$.

As a consequence of the lemma and the table of ancipital forms, we may deduce the following theorem.

Theorem 4. (a) Let $p \equiv q \equiv 1(\bmod 8)$ and $(p \mid q)=1$. Then all four ambiguous classes are in $G^{+}$, so $b^{\prime}(8 p q) \equiv 0(\bmod 16)$. Write $p q=2 f^{2}-e^{2}=2 b^{2}+a^{2}$ with $a \equiv e \equiv 1(\bmod 4)$. If $(a \mid p)=(a \mid q)=(e \mid p)=(e \mid q)=1$, then $b^{\prime}(8 p q) \equiv$ $0(\bmod 32)$. If each of the following conditions bolds then $N \epsilon=-1$ and $b(8 p q)=$ $b^{\prime}(8 p q) \equiv 16(\bmod 32):$ 
(i) $(a \mid p)=-1$ or $(a \mid q)=-1$;

(ii) $(e \mid p)=-1$ or $(e \mid q)=-1$;

(iii) $(2 \mid p)_{4} \neq(q \mid p)_{4}$;

(iv) $(2 \mid q)_{4} \neq(p \mid q)_{4}$;

(v) $(p \mid q)_{4}=(-1)^{(p+7) / 8}$; and

(vi) $(q \mid p)_{4}=(-1)^{(q+7) / 8}$.

Otherwise, we have no information.

(b) Let $p \equiv q \equiv 1(\bmod 8)$ and $(p \mid q)=-1$; write $p q=2 f^{2}-e^{2}=2 b^{2}+a^{2}$ with $a \equiv e \equiv 1(\bmod 4)$. If $(a \mid p)=(a \mid q)=(e \mid p)=(e \mid q)=1$, then $b^{\prime}(8 p q) \equiv$ $0(\bmod 16) ;$ otherwise, $b^{\prime}(8 p q) \equiv 8(\bmod 16)$. If (i) $(a \mid p)=-1$ or $(a \mid q)=-1$ and (ii) $(e \mid p)=-1$ or $(e \mid q)=-1$, then $N \epsilon=-1$ and $b(8 p q)=b^{\prime}(8 p q) \equiv 8(\bmod 16)$. Otherwise, $b^{\prime}(8 p q) \equiv 0(\bmod 8)$.

(c) Let $p \equiv 1$ and $q \equiv 3(q \equiv 7)(\bmod 8)$ and $(p \mid q)=1$; write $p q=2 f^{2}+$ $e^{2}\left(2 f^{2}-e^{2}\right)$ with $e \equiv 1(\bmod 4)$. Then $N \epsilon=1$. If $(e \mid p)=-1$ or $(e \mid q)=-1$ or $(2 \mid p)_{4} \neq(q \mid p)_{4}$, then $b^{\prime}(8 p q) \equiv 8(\bmod 16) ;$ if $f_{1} \sim f_{p}$ or $f_{1} \sim f_{q}\left(f_{1} \sim f_{p}\right.$ or $\left.f_{1} \sim f_{-q}\right)$ and if $(e \mid p)=(e \mid q)=1$, then $b^{\prime}(8 p q) \equiv 0(\bmod 16)$. Otherwise we have no information, except that $b^{\prime}(8 p q) \equiv 0(\bmod 8)$.

(d) Let $p \equiv 1, q \equiv 5(\bmod 8)$ and $(p \mid q)=1$. If $(2 \mid p)_{4} \neq(q \mid p)_{4}$ or if $(p \mid q)_{4}=$ $(-1)^{(p+7) / 8}$, then $b^{\prime}(8 p q) \equiv 8(\bmod 16) ;$ if both of these conditions occur, then $N \epsilon=-1$ and $b(8 p q) \equiv 8(\bmod 16)$. If neither occurs, then $b^{\prime}(8 p q) \equiv 0(\bmod 8)$.

(e) Let $p \equiv q \equiv 7(\bmod 8) ;$ then $N \epsilon=1$ and $b^{\prime}(8 p q) \equiv 0(\bmod 8)$.

The proof of this theorem relies on group-theoretic arguments similar to those used in the proofs of previous theorems. Conditions (a)(i) through (a)(vi) imply that the only ancipital forms which are fourth powers are $f_{1}$ and $f_{-1}$; hence $f_{1} \sim f_{-1}$ and $N \epsilon=-1$. The other statements are easily verified, so we omit the remainder of the proof. Within the limitations of our techniques, it appears that our results are best possible.

8. The case $d=p_{1} p_{2} p_{3}, p_{i}$ odd primes. Since $d \equiv 1(\bmod 4)$ we may assume that $p_{1} \equiv 1$ and $p_{2} \equiv p_{3}(\bmod 4)$. We will simply state the results, as the proofs are obtained in precisely the same manner as were the previous proofs.

Theorem 5. (a) Let $p_{1} \equiv 1$ and $p_{2} \equiv p_{3} \equiv 3(\bmod 4)$. Then $N \epsilon=1$ and $b^{\prime}\left(p_{1} p_{2} p_{3}\right) \equiv 0$ or $4(\bmod 8)$ according as $\left(p_{1} \mid p_{2}\right)=\left(p_{1} \mid p_{3}\right)=1$ or not. If $\left(p_{1} \mid p_{2}\right)=\left(p_{1} \mid p_{3}\right)=1$ and $\left(p_{2} \mid p_{1}\right)_{4} \neq\left(p_{3} \mid p_{1}\right)_{4}$, then $b^{\prime}\left(p_{1} p_{2} p_{3}\right) \equiv 8(\bmod 16)$; otherwise we have no information.

(b) Let $p_{i} \equiv 1(\bmod 4), i=1,2,3$. Then $b^{\prime}\left(p_{1} p_{2} p_{3}\right) \equiv 0$ or $4(\bmod 8)$ according as at most one or at least two of the symbols $\left\{\left(p_{1} \mid p_{2}\right),\left(p_{1} \mid p_{3}\right),\left(p_{2} \mid p_{3}\right)\right\}$ equal -1 ; if they are all equal to 1 , then $b^{\prime}\left(p_{1} p_{2} p_{3}\right) \equiv 0(\bmod 16)$.

(c) Let $p_{i} \equiv 1(\bmod 4), i=1,2,3,\left(p_{1} \mid p_{2}\right)=\left(p_{1} \mid p_{3}\right)=1$ and $\left(p_{2} \mid p_{3}\right)=-1$. If either $\left(p_{2} \mid p_{1}\right)_{4} \neq\left(p_{3} \mid p_{1}\right)_{4}$ or $\left(p_{1} \mid p_{2}\right)_{4} \neq\left(p_{1} \mid p_{3}\right)_{4}$ then $b^{\prime}\left(p_{1} p_{2} p_{3}\right) \equiv 8(\bmod 16)$, 
and if both relations bold, then $N \epsilon=-1$ and $b\left(p_{1} p_{2} p_{3}\right) \equiv 8(\bmod 16)$. Otherwise we bave no information.

(d) Let $p_{i} \equiv 1(\bmod 4), i=1,2,3$ and $\left(p_{i} \mid p_{j}\right)=1$ for $i \neq j$. If $\left(p_{i} \mid p_{j}\right)_{4}\left(p_{i} \mid p_{k}\right)_{4}=$ $\left(p_{j} \mid p_{i}\right)_{4}\left(p_{k} \mid p_{i}\right)_{4}=-1$ for $i, j$ and $k$ distinct, then $b^{\prime}\left(p_{1} p_{2} p_{3}\right) \equiv 16(\bmod 32)$ and $N \epsilon=-1$. Otherwise we bave no further information.

Remark. The difficulty in obtaining further information in Theorems 4 and 5 stems from the fact that certain given conditions on fourth power residue symbols are necessary, but not sufficient, for certain ambiguous classes to be fourth power.

9. A problem. The techniques of this paper have been used to treat imaginary fields with two or three divisors of the discriminant [1], [3] and clearly may be used to treat arbitrary quadratic fields. In each of the theorems of this and previous papers on the subject, there are open cases. What can be said about these cases?

\section{REFERENCES}

1. Ezra Brown, The class number of $Q(\sqrt{-} p)$ for $p \equiv 1(\bmod 8)$ a prime, Proc. Amer. Math. Soc. 31 (1972), 381-383. MR 44 \#6645.

2. - Binary quadratic forms of determinant - pq, J. Number Theory 4 (1972), 408-410. MR $46 \# 134$.

3. - Class numbers of complex quadratic fields, J. Number Theory (to appear).

4. Harvey Cohn, A second course in number theory, Wiley, New York, 1962. MR 24 \# A3115.

5. Burton W. Jones, The arithmetic theory of quadratic forms, Carus Monograph Series, no. 10, The Math. Assoc. of America, Buffalo, N. Y., 1950. MR 12, 244.

6. Gordon Pall, Binary quadratic discriminants differing by square factors, Amer. J. Math. 57 (1935), 789-799.

7. - Discriminantal divisors of binary quadratic forms, J. Number Theory 1 (1969), 525-533. MR 40 \#1335.

DEPARTMENT OF MATHEMATICS, VIRGINIA POLYTECHNIC INSTITUTE AND STATE UNIVERSITY, BLACKSBURG, VIRGINIA 24061 